\title{
Contention-Aware Spectrum Sensing and Access Algorithm of Cognitive Network
}

\author{
Invited Paper
}

\author{
HU Gang \\ Computer School of National University of Defense \\ Technology, Changsha, China \\ golfhg@vip.sohu.net, xuming-64@hotmail.com
}

\author{
ZHANG Qian*, XU Ming \\ Computer Science and Engineering of HKUST \\ HONGKONG, China, * \\ qianzhang@cse.ust.hk
}

\begin{abstract}
The technology of sensing and access the potential available spectrum is always a hot topic for cognitive radio networks. Compare to the existing works, this paper proposes a new centralized sensing and access protocol which is based on the premise of contention-aware network flow. The target of this protocol is to maximize the expected throughput of the whole cognitive network. We have proven that finding the optimal sensing and access results under a fixed contention topology is NP problem. An approximated algorithm is proposed which is consisted by minimum clique cover and maximum matching algorithm. By detailed simulation, the performance of the approximated algorithm have at least $30 \%$ improvement comparing to the optimal sensing and access scheme which do not adopt the clique cover and maximum matching scheme.
\end{abstract}

\section{Keywords-spectrum;sensing;access;contention-aware}

\section{INTRODUCTION}

As the rapid development of wireless devices becoming more and more prevalent, the current fixed spectrum policy can't meet the increasing need of spectrum utilization. From the investigation of [1], the fact is that a large portion of the assigned spectrum is used sporadically and geographical variations in the utilization of assigned spectrum ranges from $15 \%$ to $85 \%$ with a high variance in time and space. The paradox indicates that the spectrum shortage is not true in physical world. The bottleneck is the long time existed spectrum usage policy.

It is impossible to change all the existing spectrum management policies at once. One practical approach is to utilize the spectrum resource more efficiently without interfere with the licensed users. The cognitive radio may make it become reality. As cognitive radio is first promoted at the end of 20th century, it has now been widely regarded as the foundation as next generation of wireless network. Now it has driven many research works focuses on the area of cognitive radio and open spectrum network [2].

We consider a cognitive network consists of two types of users. One kind is primary user and another is secondary user. The core advantage of cognitive radio is that it can dynamic sense and access the blank spectrum-holes which are not used by primary users during a certain period of time. The spectrum's iterative using by two types of users can efficiently improve the spectrum utilization. Thus, how to sense and access the blank spectrum is one of the key issues of cognitive network.

There're many existing works which are focused on the spectrum sensing and access technique for a specific open spectrum environment. In [3], a cooperative algorithm based on interference temperature model is proposed for the computation of available channels. It focuses on the occupancy and availability of one certain channel. An optimal opportunistic spectrum access policy is proposed for determining which channel to probe and which channel for transmission [4]. It has proved that the optimal strategy has threshold property. Similar work of optimal sensing and access strategy can be found in [5], which models the problem into an optimal stopping problem, a multi-band opportunistic auto rate protocol named MOAR is designed to improve the throughput. Another kind of work which is based on markov transition model can be found in [6,7]. [6] develops an analytical framework for opportunistic spectrum access based on the theory of partially observable markov decision process. It proposed a decentralized MAC protocol, and take sensing error into consideration, the main deficiency of the paper is the assumption of state transition probabilities are all known to secondary user is not practical. The main difference of [7] is using a primary-prioritized markov approach to model the interactions between the primary users and the unlicensed users as continuous-time markov chain. Another latest work on optimal sensing and access is the HC-MAC[8] protocol, which take hardware constraints as the main criterion when consider the sense and access scheme, an optimal stopping model is used to formulate the tradeoff between the sense and access process, and a decentralized MAC protocol is proposed for the ad hoc cognitive network.

All the above works are using different models (for example, markov model, optimal skipping model, etc.) to formulate the specific sensing and access process, they can find the optimal available channels to sense or to access under a certain open spectrum environment. Except [5,8], most of the existing works do not consider the hardware constraints of the sensing process. The most deficiency of the above works is that they don't take the network's contention relation into consideration when prosecute the sensing and access process. As the potential interference exists between the contending links, the optimal access scheme will lead to a certain degree 
of performance descending. Another important disadvantage of not taking contention relation into considering is to influence the efficiency of sensing decision. Because the sensing process may waste time to sense the potential contention channels which will be used simultaneously. So, we need to taking contention relation into consider when designing the sensing and access algorithm. So in this paper, a new joint sensing and access algorithm is proposed which can efficiently adapt the traffic flow's contention relation of the network.

There're many challenges in the design of spectrum sensing and access strategy. First, it is challenging to identify the temporal blank spectrum quickly and accurately. Second, how to access the blank spectrum is a challenging problem as the secondary user should guarantee the non-interference access with primary users and maximize utilizing the accessible spectrum. Our work not only considers the above two aspects, but also considers the traffic of secondary users. It is much more difficult to design the sensing and access protocol when considering the contention relation between different traffic flows. Because the common optimal sensing and access scheme just find the optimal result from the perspective of channel's status, our problem will combine the channel's status and network topology into consideration. It will lead the computer complexity to increase exponentially as the contending node's increase. The result for each individual node may not be the best, but from the network perspective, it will lead to an optimal performance.

The main target of this paper is to find the sensing and access channels of each node so that they can lead to the optimal expected network throughput. The main idea of the paper is to using a new clique cover algorithm to model the contention relation of the whole network, so the problem is transformed to a network's maximal matching of the transformed bipartite graph. After the maximal matching process, we can get the near-optimal result for the contentionaware sensing and access problem

The contribution of this paper can be concluded as this:

1) We mathematically formulated the problem of contention-aware spectrum sensing and access into a $\mathrm{K}-\mathrm{CSP}$ problem, and proved it is NP problem.

2) A novel approximated centralized sensing and access algorithm which is based on minimum clique cover algorithm and maximum matching algorithm is proposed.

3) Through detailed simulation, we can see the approximated centralized algorithm can improve the expected throughput efficiently.

The organization of this paper is: section II is the problem formulation, which have four sections: section $\mathrm{A}$ is the network model's description, section B explains the motivation for our this paper, section $\mathrm{C}$ and $\mathrm{D}$ are the mathematical expressions of the problem and the NP prove process; the algorithm is detailed expressed in section III; simulation results and is in section IV. The last section is the conclusion and the references of this paper.

\section{PROBLEM FormULATION}

\section{A. Network Model}

Given a wireless cognitive network which consists of $N=\left\{n_{1}, n_{2}, \ldots n_{n}\right\}$ secondary users and several primary users, assume there're $M=\left\{c_{1}, c_{2}, \ldots, c_{m}\right\}$ available channels which are shared by both primary and secondary users. For simplicity, we assume all the channels are equally divided and have the same bandwidth of $b(M / s)$. Each secondary user has heterogenous channels that can be sensed and accessed. All the network's available channel set can be expressed by $\varphi=\left\{\varphi_{1}, \varphi_{2}, \ldots \varphi_{n}\right\}, \forall \varphi_{i \in[1, \ldots, n]} \in M$.

Each secondary user has two different radios. One is cognitive radio, another is common wireless radio. All the secondary users use this common radio on a specific control channel. The function of this common channel is to provide a unified system clock for all the secondary users. The control packets are also transmitted by this common channel.

One of the major advantages of cognitive radio is the capability of aggregating several spectrums for transmission. No matter the spectrums are consecutive or separated in the frequency range. But the width and number of channels are limited as the hardware limitation, the detailed content can be found in [9].

TABLE 1 SYMBOL EXPRESS

\begin{tabular}{|c|c|}
\hline$N$ & the whole number of secondary users. \\
\hline$M$ & $\begin{array}{l}\text { The whole spectrum which is divided equally and seen } \\
\text { by all users. }\end{array}$ \\
\hline$F$ & All the network flows which have fixed routing path. \\
\hline$T$ & A fixed sensing and access period. \\
\hline$\Delta t$ & The fixed time of one channel's sensing process. \\
\hline$t_{a}$ & The time of access after sensing period has finished. \\
\hline$C_{i j}$ & The contention relation between link $i$ and $j$. \\
\hline$p$ & $\begin{array}{l}\text { The probability of primary user's traffic which does not } \\
\text { occupy the channel during each } T \text {. }\end{array}$ \\
\hline$R_{t}$ & The transmitting range \\
\hline$R_{i}$ & The interference range \\
\hline$d(i, j)$ & The distance between two links $l_{t}$ and $l_{f}$ \\
\hline$C_{I}(i j)$ & $\begin{array}{l}\text { The number of contention links which will cause } \\
\text { interference with } f_{i j}\end{array}$ \\
\hline$b$ & The bandwidth of a channel \\
\hline$C Q_{i}$ & clique $i$ \\
\hline
\end{tabular}

The operations of secondary devices usually have two stages: sensing and transmission (or access). As the hardware constraints of the cognitive radio, the sensing process needs a 
certain period of time and some energy to accurately evaluate the channel's status. For simplicity, here we just take time cost into consideration. The access period is to using the optimal channels for transmitting. Another important assumption is that the sensing process will not interfere with other users' transmission.

The draft of $802.22[10]$ has defined the primary user's maximum tolerating time when the licensed channel is occupied by secondary user. It means no matter what the channel condition is, the secondary user must work periodically within fixed maximum time. Here we consider the fixed period time for a round of spectrum sensing and access process. The process is sequentially proceeding as fig 1 . During a fixed transmitting period, the time slots is like $\left\{\Delta t_{1}, \Delta t_{2}, \ldots, \Delta t_{k}, T-k \cdot \Delta t\right\}$, the first part is the sensing process, the last part is access period. For simplicity, we consider the channels' sensing cost is the same $\Delta t$. The whole target of this algorithm is to minimize the sensing process and maximizing the access period of the entire network.

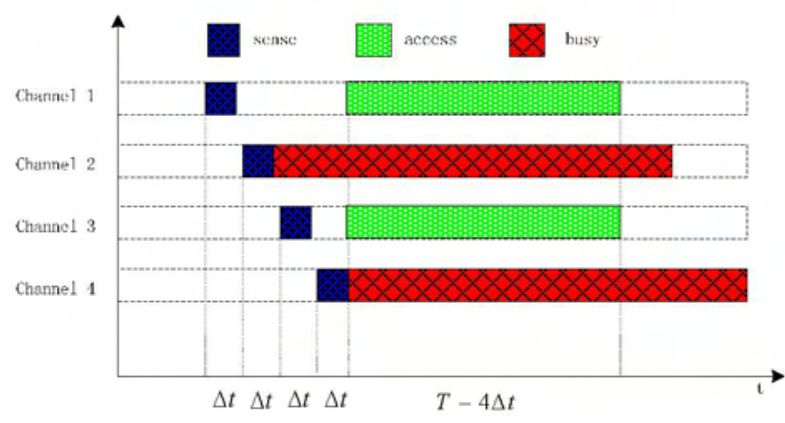

Fig1 the sensing and access process

\section{B. Motivated Scenario}

Now we take a simple network into consideration. Assume there're four secondary users, which is constructed into two contending flows, as seen in fig 2. Each contending flow is within another's interference range. So, the two flows will lead to collision if they using a common channel to transmit simultaneously. For the existing work, each flow will try to maximize its own access bandwidth. The greedy strategy is to sensing all the accessible channels within its sensing capacity and accessing the optimal channels. Given the status of the four channels of one period in table 2, the optimal algorithm may find the all available channels to sense, for example, $n_{1}$ will find the channel $\left\{c_{1}, c_{2}, c_{3}, c_{5}\right\}$ to sense, the optimal access channels of the network is: $F_{1}$ using channel $c_{1}$ and $c_{2}$; $F_{2}$ using channel $c_{3}$ and $c_{4}$. Considering the sensing overhead, the actual network's throughput which can be calculated as $4 * b *\left(1-\frac{4 \Delta t}{T}\right)$.

Now if we know the contention relation exists between the two flows, then we can adjust the sensing and access strategy to improve the throughput. The optimal sensing set for flow $n_{1}-n_{2}$ is $\phi_{1}=\left\{c_{1}, c_{2}\right\}$, and flow $n_{3}-n_{4}$ sensing set is $\phi_{2}=\left\{c_{3}, c_{4}\right\}$. And the optimal access scheme is the same as table 2. Compare to the original method, the throughput will

\footnotetext{
Supported by NSF of China, No 60773017 .
}

be improved to $4^{*} b^{*}\left(1-\frac{2 \Delta t}{T}\right)$, if we set $\frac{\Delta t}{T}=0.1$, the throughput improvement will be $33.3 \%$. The result is shown in fig 3 .

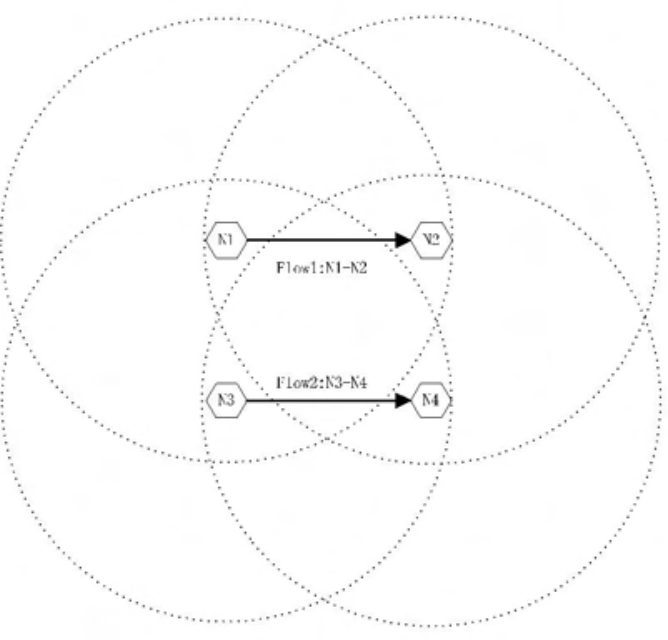

Fig2 the contending flow topology

TABLE 2 THE PRACTICAL CHANNEL USABILITY

\begin{tabular}{|l|l|l|l|l|l|l|}
\hline & $c_{1}$ & $c_{2}$ & $c_{3}$ & $c_{4}$ & $c_{5}$ & access \\
\hline$n_{1}$ & 1 & 1 & 1 & 0 & 1 & $c_{1}+c_{2}$ \\
\hline$n_{2}$ & 1 & 1 & 0 & 1 & 0 & $c_{1}+c_{2}$ \\
\hline$n_{3}$ & 0 & 1 & 1 & 1 & 1 & $c_{3}+c_{4}$ \\
\hline$n_{4}$ & 1 & 0 & 1 & 1 & 0 & $c_{3}+c_{4}$ \\
\hline
\end{tabular}

In practical, it is not realistic to get the optimal result from the start as we don't have the practical channel status for any moment. As each channel's status can just be determined after the sensing process, it is impossible to find the optimal results before the sensing process. But if we assume each channel's availability is the same $\mathrm{p}$, we can get a near-optimal result.

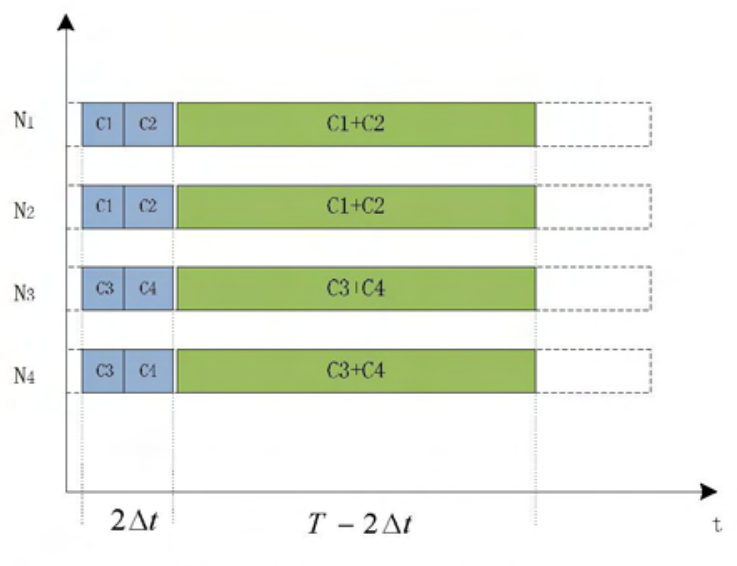

Fig 3 an optimal result of sensing and access

\section{Mathematical Expression}

We assume the whole network is working under a timeslotted way. The period is fixed to $T$ for one round of spectrum sensing and access. The sensing process must be finished before access process start. The more complicated 
scenario is to design the two processes randomly, which means after each time slot, the node will make a decision to sense or access. This scenario is out of the scope of this paper. So, during a certain period $T$, the time slots are represented like $\left\{\Delta t_{1}, \Delta t_{2}, \ldots, \Delta t_{k}, T-k \cdot \Delta t\right\}$, here $\Delta t_{k}$ is the $k$ th sensing time for a candidate channel. For simplicity, we take each channel's sensing time is identical as $\Delta t$.

Besides each secondary user knows its own accessible channel set from the $\varphi=\left\{\varphi_{1}, \varphi_{2}, \ldots \varphi_{n}\right\}, \forall \varphi_{i \in[1, \ldots, n]} \in M$, another premise of the traffic flows $F=\left\{F_{1}, F_{2}, \ldots, F_{F}\right\}$ are known for all the secondary users too. Here we do not constrain the maximum flow throughput to a fixed value, the secondary user may use as much as possible aggregated channels for access within its hardware constraints. Although different cognitive radios may have different capability, we just consider the situation that all the secondary users have the same kind of cognitive radio which has the same capacity of aggregated channels. For a given traffic flow, the routing path is known and fixed. If the routing path is $F=\left\{n_{1}, n_{2}, \ldots, n_{i}\right\}$, we use the sub-flow to represent the links along the path. So that $F_{t}=\left\{f_{1,2}(i), \ldots, f_{(j-1), j}(i)\right\}$.

As the contention relation is known to all the secondary user, the definition of contention here is from a typical protocol model. In this protocol model, the transmission from node $n_{i}$ to $n_{j}(i, j \in N)$ is successful if: 1) the distance between these two nodes $d_{i j}$ satisfies $\left.d_{i j}<d_{t x} ; 2\right)$ any node $n_{k}(k \in N)$, which is within the interference range of the node $n_{i}$ and $n_{j}$ (i.e., $d_{k j}<d_{\text {int }}$ or $d_{k i}<d_{\text {int }}$ ) is not transmitting. So, based on the model, we define the flow contention in a location dependent manner: two sub-flows contend with each other if either the source or destination of one sub-flow is within the interference range of the source or destination of the other. So, all the sub-flows' contention relation can be expressed as a contention matrix of

$$
C=\left(\begin{array}{ccc}
\left\{f_{1,1}\right\} & \cdots\left\{f_{1, j}\right\} \cdots & \left\{f_{1, n}\right\} \\
\vdots & \ddots & \vdots \\
\left\{f_{n, 1}\right\} & \cdots\left\{f_{n, j}\right\} \cdots & \left\{f_{n, n}\right\}
\end{array}\right)
$$

In this matrix, each $\left\{f_{i j}\right\}$ represents the set of its contending sub-flows. We define the value of the number of the set $\left\{f_{i j}\right\}$ as the contention index--- $C_{I}\left(f_{i j}\right)$.

As the sensing process need time to execute, there exist a tradeoff between the sensing and access. We define the efficient throughput to reflect the tradeoff:

$$
T h\left(f_{v}\right)=\frac{\Delta g * b^{*}(T-k * \Delta t)}{T * C\left(f_{i}\right)}
$$

In this formula, $\Delta g$ is the number of aggregated accessible channels, and $k$ is the number of sensed channels before access. Now the problem is: Given the channel set $\varphi$ and contention matrix $C$, how to design a joint sensing and access strategy, which can maximum the whole network's expected throughput? It can be formulated as to:

$$
\max \sum_{F_{i}} \min \left(T h\left(F_{i}\right)\right)
$$

The result of the strategy is to provide each node a sensing set $\left\{\phi\left(n_{i}\right)\right\}$ and the access channels for transmitting, which can lead to the maximum network flows' expected throughput.

\section{NP prove process}

Now we construct a special instance of our problem, and prove that it is equivalent to the MAX k-CSP problem.

1) We set $\{U\}=\{M\}$; each channel represents each variable;

2) We set each $\left\{S_{i}\right\}=\left\{C_{i}\right\}=\left\{c_{1} \vee c_{2} \vee \ldots \vee c_{k}\right\}$;

3) Each channel's value is assigned as $c=\{0,1\}$, which is equal as each literal's assigned value of $\{U\}$;

4) The network's flow establishment must satisfy the requirement of common channel, which can be represented as $C=\left\{C_{1} \wedge C_{2} \wedge \ldots \wedge C_{N}\right\},\left\{N^{\prime}\right\} \in\{N\}$, which are nodes that have traffic to send and receive parallel.

Now the problem is to find a maximum $\mathrm{k}$ literal of each clause's assignment function $f(U)$, which can satisfy the

$$
C=1 \text {. }
$$

Two necessary conditions must be emphasized:

1. The value of cognitive radio network's channel is not consistently seen by all users, here we can assume the nodes of $\left\{N^{\prime}\right\}$ have the consistent view of their channels, which means the value of channel $c_{t}$ is the same to all nodes.

2. We take the parallel sub-flow's transmitting as a necessary condition, which guarantee all the nodes will not always transmit in turn.

As the MAX k-CSP is a well-known NP problem, it is easy to know the special instance we constructed can't be done within poly-nominal time. Now the complexity of function $f(U)$ is the same as the channel assignment algorithm.

\section{APProximated CENTRAlized Algorithm}

As we have proven finding a satisfied solution of our problem is NP problem, now we want to find an approximate approach. The purpose of the algorithm is to find a nearoptimal results which can be found within poly-nominal time. And it can provide a reference for the protocol design in the future.

The motivation of the algorithm is classical philosophy --"divide and conquer". Because the ideal optimal results can only be found by searching all the combinations of the result space, it naturally causes us to divide the whole network into several parts. Here, we use the minimum clique cover algorithm to prosecute the "divide" part. And the "conquer" 
part is to model the clique into bipartite graph, through maximize matching algorithm, we can get the optimal results of each clique, then we get the whole network's near-optimal results.

\section{A. The Clique Cover Algorithm}

The clique cover algorithm is to get an approximated division of the contention graph [11]. After the division, each link is classified to a certain clique. There're three types of links: one is connected neighbor link, the second is no contenting parallel link and the third is parallel contention link. Intuitively, we need to calculate each link's sensing results within all the other links' relation to this link. But the clique cover algorithm can reduce the compute complexity. The algorithm is to find a minimum number of cliques which can cover the whole network nodes. As the minimum clique cover algorithm is also a NP problem, we designed a poly-nominal approximated algorithm to find the minimum results.

\section{Algorithm 1(clique cover of network):}

Input: Graph $G=(\{1,2, \ldots, n\}, E)$

Output: A minimum cardinality clique cover for $G$

1. Initialize the graph:

1.1 If distance $(i, j)<R_{i}, f_{i j}=1$; set CliqueofSize $(2)=[i, j]$;

else $f_{i j}=0$;

endif;

1.2 Find the separated nodes;

2. While CliqueofSize $(K) \neq \phi$; Find all the CliqueofSize $(K)$;

3. If Cliqueofize $(K)=\phi$; Find the minimum clique cover from the calculated cliques from Cliqueofsize(2) to CliqueofSize $(K)$; else, switch to step2. endif;

4. Deleting the overlapped cliques

4.1 If can't delete any one clique for all the nodes, stop. endif

5. Output the all the cliques;

Fig 4 is a clique covering result. The contention graph is covered by two cliques, which guarantees each node belongs to at least one clique.

\section{Capacity Analysis:}

After the above algorithm, all the nodes are covered by at least one clique. Based on the clique cover result, the whole network's capacity can be calculated:

Maximum parallel throughput:

$$
\begin{gathered}
T h_{\max }=\sum_{i=1}^{i=k} \operatorname{Th}\left(C Q_{i}\right) \\
T h\left(C Q_{i}\right)=\max \left\{f_{p q}\right\}, \forall f_{p q} \in C Q_{i}
\end{gathered}
$$

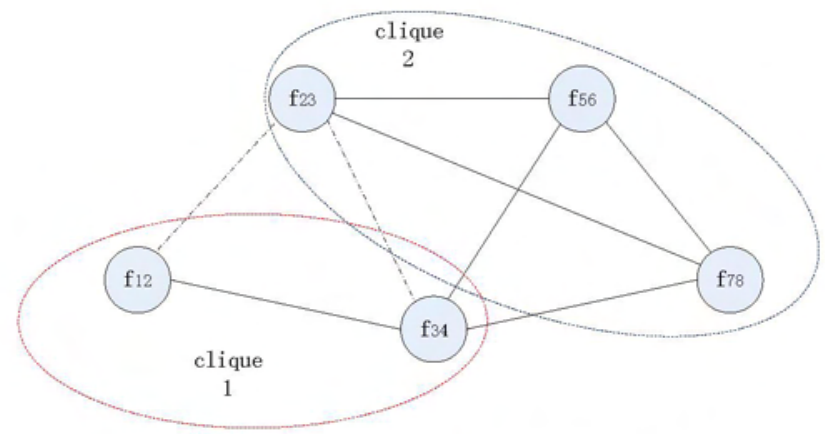

Fig 4 a clique cover algorithm result example

The maximum parallel throughput can be achieved only when each clique chooses the maximum sub-flow node which is covered by only one clique. It is more practical to take all the nodes into calculation. Taking fairness into consideration, we assume each sub-flow node is equally scheduled within a period of transmitting time. Then the network's throughput can be calculated as:

$$
T h=\sum_{i=1}^{i=k} \operatorname{Th}\left(C Q_{i}\right)-\sum_{i=1}^{i=k} \operatorname{Th}\left(C T_{i}\right)
$$

The above equation is not a fixed value as $\sum_{t=1}^{t=k} T h\left(C T_{t}\right)$ is determined by different scheduling schemes. We assume the schedule is perfect and can achieve the maximum throughput.

From the equation above, we can see there're two approaches to improve the capacity: one is to increase the clique cover number of the whole network; the other is to decrease the constraints of sub-flow nodes which are covered by multiple cliques. And the two approaches are intrinsically the same.

\section{B. Sensing Decision Algorithm}

The sensing set algorithm is to calculate each sub-flow should sense how many channels and what are these channels.

What is different from traditional channel assignment is the channel needs to be sensed before access. Each channel has a probability to access as the existence of primary user. Another fundamental difference is the cognitive radio has certain hardware constraints, which means we want to sense the channel more efficiently because each channel's sensing costs time. And each node can aggregate a limited maximum number of channels for transmitting.

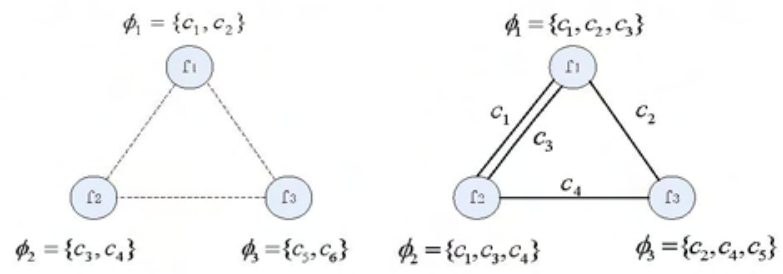

Fig 5 two examples of sensing set allocation within a clique

In the left sub-graph of fig 5, each node has two candidate channels to be sensed. 
Here the challenging problem we should consider is: what is the optimal tradeoff between increasing the parallel links and the aggregated link capacity.

There exist three elements which influence the tradeoff: One is the maximum number of aggregated channels. Second is the ratio between each node's channel subset and the whole channel set. Third is the similarity extent between contention links.

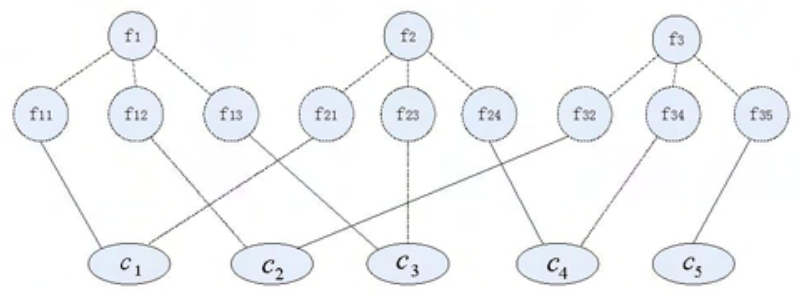

Fig 6 the bipartite graph

The sensing set algorithm inside an isolated clique can be mathematically represented by a bipartite graph, the two node sets are node set and channel set of a clique. Which can be represented by $X=\left\{N_{i}\right\}, \forall N_{i} \in C Q \in N, Y=\left\{c_{j}\right\}, \forall c_{j} \in M$. The edges between two sets represent the channels of each node. So the sensing set allocation algorithm is transformed to a maximum matching of the corresponding bipartite graph. The sensing set allocation algorithm is to allocate different channel to each nodes as much as possible.

The maximum matching of a bipartite graph is defined as: Given a match of $M$, for any other matching $M^{\prime}$, there is always $\left|M^{\prime}\right|<|M|$, we call this matching $M$ is maximum matching.
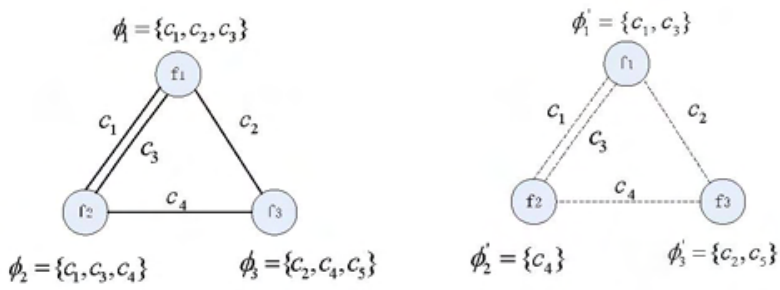

Fig 7 the maximum matching of bipartite graph

Another important aspect of our algorithm is how to deal with the nodes which are covered by multiple cliques.

According to the above scenario, we need a criterion to decide how to classify the overlapped node $f_{3}$ to which clique. We define a contention portion index as:

$$
C_{p}(i)=\frac{\sum_{i}\left(C h_{t}\right)}{\sum_{i=1, \ldots, k} \sum_{i}\left(C h_{t}\right)}
$$

The meaning of this index is to judge the portion of a clique that covers the nodes compare to all the cliques that covers the node. We then classify the overlapped node to the clique which has the minimum $C_{p}(i)$.

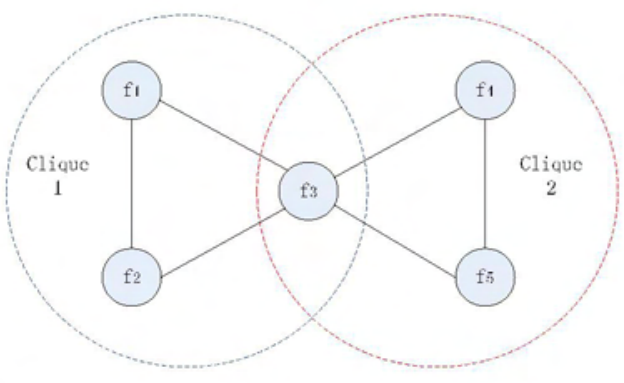

Fig 8 an example of clique cover by multiple cliques

Algorithm 2 (A maximum matching algorithm)

[To determine a maximum matching in a bipartite graph $\boldsymbol{G}$ with $V(G)=\left\{v_{1}, v_{2}, \ldots, v_{n}\right\}$ and sensing $\left.\operatorname{set} \varphi=\left\{\varphi_{1}, \varphi_{2}, \ldots, \varphi_{n}\right\}\right]$

1. Initialize: for all the contenting nodes; arrange the sequence of cliques from higher cardinality to lower cardinality;

2. If there're unmatched clique, then continue; otherwise, stop, the matching $M$ is maximum now.

3 If all the node $v$ of CliqueofSize $(k)$ is matched, $k=k-1$,return to step 2 ;

otherwise, using hungarian algorithm to $\operatorname{assign} \varphi_{i}$ to CliqueofSize $(k)$, get the result of $\varphi_{i}^{\prime}$;

4. If node is covered by multiple cardinality cliques, choosing the remaining $\varphi_{i}=\varphi_{i}-\varphi_{i}^{\prime}$, using hungarian algorithm for the new CliqueofSize $(k)$;

5. Output $\varphi=\left\{\varphi_{1}^{\prime}, \varphi_{2}^{\prime}, \ldots, \varphi_{n}^{\prime}\right\}$

\section{Access Scheme}

After the sensing process finished, the access scheme is to utilizing the potential available channels most efficiently.

As for each node, the expected throughput after sensing $k$ channels can be calculated as:

$$
\operatorname{Exp}\left[\operatorname{Th}\left(n_{i}\right)\right]=\sum_{i=0}^{i=k}\left(\frac{c_{k}^{i} * i * b *(1-p)^{i} *(T-i * \Delta t)}{T}\right)
$$

Given the whole network flow $\{F\}=\left\{F_{k}, k \in(1, \ldots f)\right\}$, we take each link $f_{i j}$ as one sub-flow of $F_{k}$, so each sub-flow's expected throughput after both nodes have sensed $k$ channels can be calculated as:

$$
\operatorname{Exp}\left[\operatorname{Th}\left(f_{i j}\right)\right]=\sum_{i=0}^{i=k}\left(\frac{c_{k}^{t} * i * b *(1-p)^{2 t} *(T-i * \Delta t)}{T}\right)
$$

For the whole network, the total throughput is:

$$
\max \sum_{f_{s}} \operatorname{Exp}\left[\operatorname{Th}\left(f_{i j}\right)\right]
$$

It can be calculated as the optimal channel assignment of multi-channel multi-radio ad hoc networks. The difference is available channels are from the sensing results. Here, we just take the maximum parallel accessible channels into consideration. 


\section{Simulation}

In this section, we present the simulation result of our approximated algorithm. As the main target of our algorithm is to promote the expected throughput, we focus on the throughput comparison under different network scenario.

In a square network area which is $1000 \mathrm{~m} \times 1000 \mathrm{~m}$, there randomly exist $\mathrm{N}$ nodes and $\mathrm{M}$ channels, the interference range is set to $350 \mathrm{~m}$, we do not consider the scenario that is densely overlapped. For example, the clique cardinality is not more than 5 .

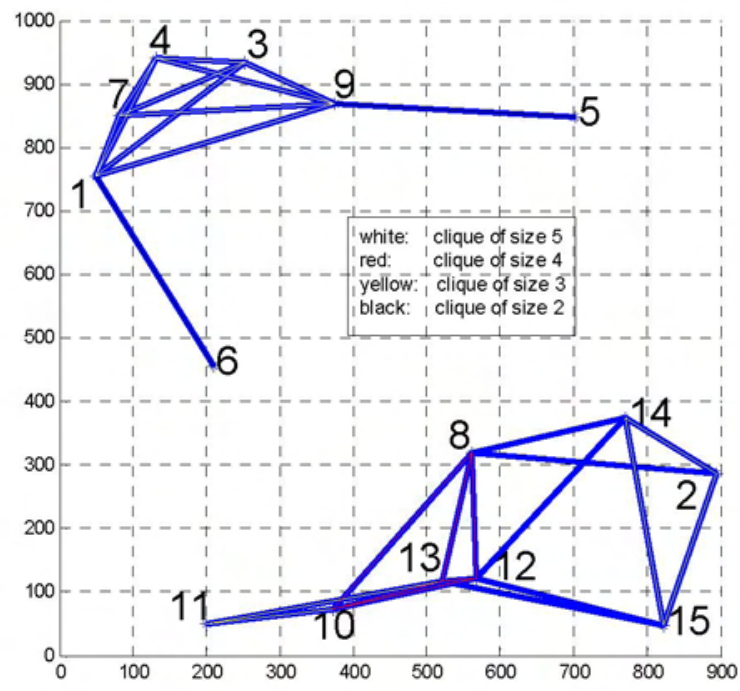

Fig 9 an example of minimum clique cover result

Figure 9 is an example of a fixed contending graph which consists by 14 contending links. We assume the cardinality of clique is 5 at most. In this graph, links $\{1,3,4,7,9\}$ are constructed as the clique of cardinality of 5 . And matching algorithm is based on the clique cover result.

Figure 10 shows the efficient utilization of a fixed 5 channels by different cardinality of clique. The upper bound of the utilization is $100 \%$ when all the channels are available and there's no sensing cost, which means $\frac{\Delta t}{T}=0$, this figure shows that as the clique size increase, the utilization will be higher, because the sensing cost is parallel distributed to each node.

The next performance result is based on the efficient use of certain available channels. The network throughput's calculation is described in the section II.

\section{A. Comparison of contending flow's number}

The most important scenario is to increasing the contending flow's number within a fixed network area. We start from 2 contending flow, then we increase each scenario by adding 2 contending flow. Here we just take 10 channels into consideration, each link's sensing set is randomly chosen 5 channels of the channel set. Assume $\frac{\Delta t}{T}=0.05$, each channel's $p=0.5$. Both throughput results are got under the

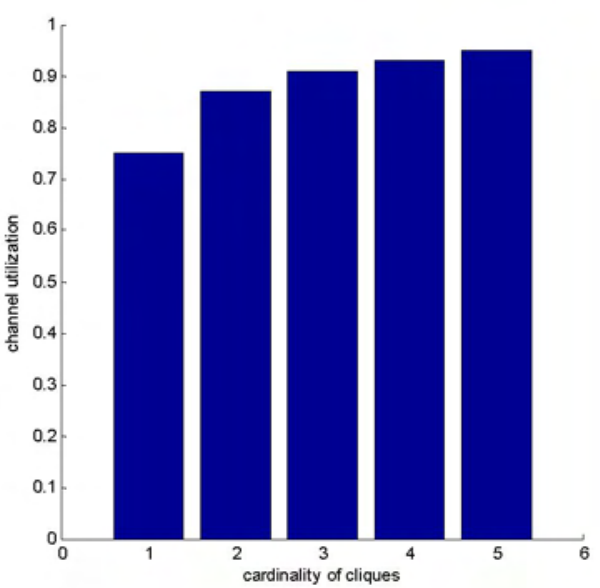

Fig 10 channel utilization of different cardinality of cliques

same network scenario. And the comparing algorithm is an optimal sensing and access scheme which do not using the clique cover and maximize matching, they just find the optimal results step by step, and this algorithm can also get the non-interference maximize accessible throughput.

The results are got from the average of 10 different topologies.

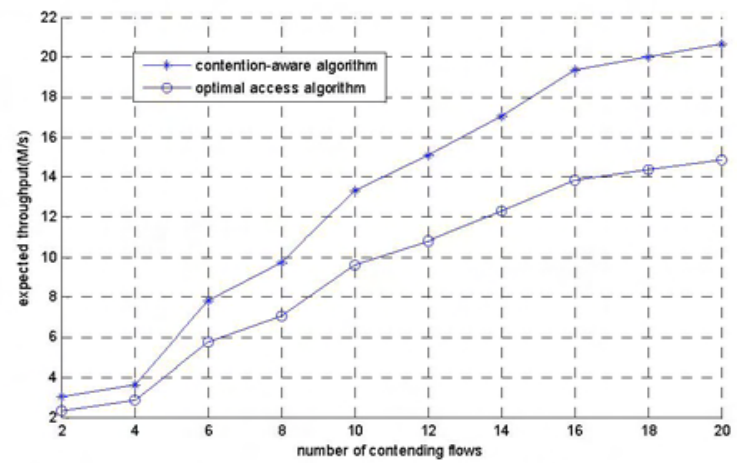

Fig 11 comparison of different contending flows

The first node is just two contending flows, here we assume the two flows are mutually contending, from the number of 4 , the contention relation is determined by the interference distance.

As we increase the number of contending flows, the throughput both improve, but the gap between the two approaches is between larger. Because as the number of contending flow's increase, the cardinality of minimum clique cover is increasing, the contention-aware algorithm can get more throughput improvement. As the contending number is between 16 and 20 , the performance improvement is much slower than the less contending scenario. As the limit of fixed available channels, the performance is restricted when the contending number increased to a certain degree.

\section{B. Comparison of different sensing set}

Another important factor that will influence the result is the sensing set. In scenario A, each link just selects 5 of the whole channels. We want to find out under a typical network topology, 
if we change the number of selected channels, what the performance we can get.

Here, we set a network of 10 contending flows, and the available sensing set is from 2 to all 10 .

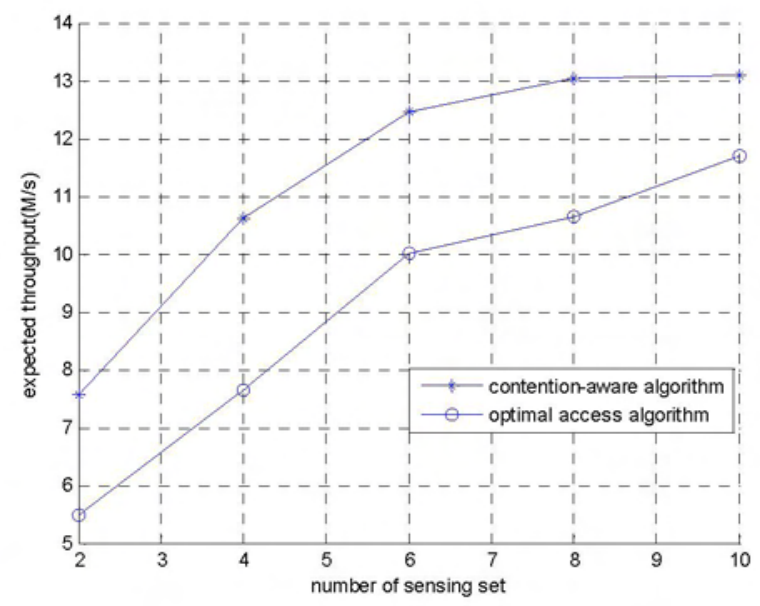

Fig 12 the comparison of different sensing set

The results are got from the average of 10 different topologies. It can be seen clearly that from the 2 to 6 sensing set, the expected throughput is linearly increased as the available channel's increase, but from 8 to 10 available channels, the performance is near to a balanced point. We found under this scenario, the performance is determined mainly by the contention relation.

\section{CONCLUSION}

In this paper, we proposed a novel problem of contentionaware spectrum sensing and access. It is proved to be NP problem. So we designed a approximate centralize algorithm to find a near-optimal results. A minimum clique cover and maximum matching algorithm is proposed to solve the problem. By detailed simulation, we can see the efficient improvement comparing to the algorithm that do not take contention relation into sensing and access decision. It can achieve at least 30\% under different network scenarios.

In the future, we want to research the scenario of different availability for each channel and heterogenous sensing set for each channel.

\section{REFERENCES}

[1] FCC, ET Docket No 03-222 Notice of proposed rule making and order, December 2003.

[2] Ian F.Akyildiz,Won-Yeol Lee,Mehmet C.Vuran,Shantidev Mohanty, "NeXt generation/dynamic spectrum access/cognitive radio wireless networks:A survey",computer networks, elsevier,50(2006) 2127-2159.

[3] Manuj Sharma,Anirudha Sahoo,K.D.Nayak."Channel Selection under Interference Temperature Model in Multi-hop Cognitive Mesh Networks", IEEE DySPAN, pages 133-136,2007.

[4] Nicholas B. Chang, Mingyan Liu,"Optimal Channel Probing and Transmission Scheduling for Opportunistic Spectrum Access", MobiCom,2007.

[5] A. Sabharwal, A. Khoshnevis, and E. Knightly. "Opportunistic spectral usage: Bounds and a multi-band CSMA/CA protocol". IEEE/ACM Transactions on Networking, pages 533-545, June 2007.

[6] Qing Zhao, Lang Tong, Ananthram Swami, and Yunxia Chen. "Decentralized Cognitive MAC for Opportunistic Spectrum Access in Ad Hoc Networks:A POMDP Framework". IEEE Journal on Selected Areas in Communications, page 589-600.April,2007.

[7] Beibei Wang, Zhu Ji, and K. J. Ray Liu. "Primary-Prioritized Markov Approach for Dynamic Spectrum Access". IEEE DySPAN 2007,page 507-515.

[8] J.Jia,Q.Zhang,and X.Shen.'HC-MAC:A Hardware-Constrained Cognitive MAC for Efficient Spectrum Management", IEEE Journal on Selected Areas in Communications, Vol.26,No.1,Janurary 2008,page:106-117

[9] A. Shukla, B.Willamson, J.Burns, E.Burbidge, A.Taylor and D. Robinson,"A Study for the Provision of Aggregation of Frequency to Provide wider Bandwidth Services", August 2006.

[10] C.Cordeiro, K. Challapali, D.Birru and S. Shankar, "IEEE 802.22: The First Worldwide Wireless Standard based on Cognitive Radios", in IEEE DySPAN 2005.

[11] Yuan Xue, Baochun Li, Klara Nahrstedt, "Optimal Resource Allocation in Wireless Ad Hoc Networks: A Price-Based Approach". IEEE Transaction on Mobile Computing, Vol.5,No.4, page 347-364, April,2006. 